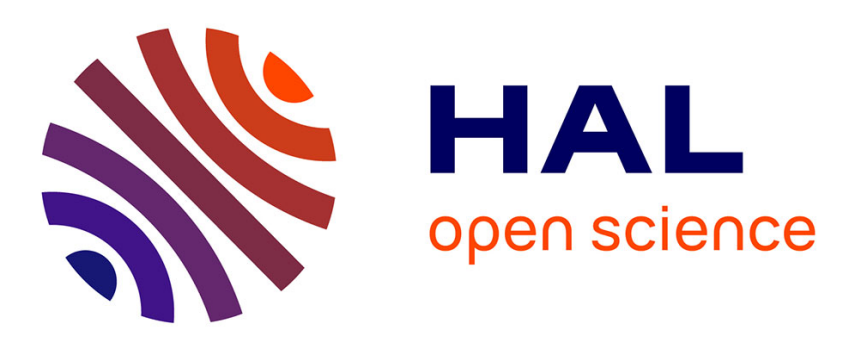

\title{
Laser induced underwater acoustic source for remote sea-bed sensing
}

\author{
S. Egerev, A. Ivakin, O. Ovchinnicov, A. Pashin
}

\section{To cite this version:}

S. Egerev, A. Ivakin, O. Ovchinnicov, A. Pashin. Laser induced underwater acoustic source for remote sea-bed sensing. Journal de Physique IV Proceedings, 1994, 04 (C5), pp.C5-351-C5-354. 10.1051/jp4:1994573 . jpa-00253068

\section{HAL Id: jpa-00253068 https://hal.science/jpa-00253068}

Submitted on 1 Jan 1994

HAL is a multi-disciplinary open access archive for the deposit and dissemination of scientific research documents, whether they are published or not. The documents may come from teaching and research institutions in France or abroad, or from public or private research centers.
L'archive ouverte pluridisciplinaire HAL, est destinée au dépôt et à la diffusion de documents scientifiques de niveau recherche, publiés ou non, émanant des établissements d'enseignement et de recherche français ou étrangers, des laboratoires publics ou privés. 


\title{
Laser induced underwater acoustic source for remote sea-bed sensing
}

\author{
S.V. EGEREV, A.N. IVAKIN, O.B. OVCHINNICOV and A.E. PASHIN
}

N.N. Andreev Acoustics Institute, 4 Shvernik Street, 117036 Moscow, Russia

\begin{abstract}
The remotely driven by means of $\mathrm{CO}_{2}$-laser pulse underwater near-surface optoacoustic source can be used for ocean medium monitoring. In the present paper this "unusual" pulse underwater signal was used for remote sea-bed sensing in shallow water regions of the Black Sea. Ultra wide band sounding pulse provided a set of narrow band reverberation responses. An appropriate sea-bed scattering model is developed provided consistence of calculated reverberation with experimental data and estimation of some sea-bed parameters as well.
\end{abstract}

\section{INTRODUCTION}

Acoustical radiation accompanying the interaction of light beam with matter can be used to solve problems in diagnostics. Diagnostic applications have been found for two mechanisms of optical sound generation, thermooptical and explosive. The latter appears to be more attractive for oceanology studies. An explosive laser induced underwater acoustic source (LIUAS), i.e., the zone of interaction of laser pulse with water surface is usually created by pulsed infrared radiation $(10.6 \mu \mathrm{m})$ from a $\mathrm{CO}_{2}$-laser when a definite threshold condition is met. Preliminary studies of LIUAS applicability to oceanology were carried out in Eighties [1]. In this paper for the first time LIUAS was used for remote sea-bed sensing.

\section{LASER INDUCED UNDERWATER ACOUSTIC SOURCE}

Suppose that $\mathrm{CO}_{2}$-laser pulse irradiates a spot of the radius $a$ at the water surface. The whole pulse is 5-10 $\mu \mathrm{S}$ width. Optical energy is absorbed by a thin $(0.001 \mathrm{~cm})$ surface layer. At small energy deposits thermal expansion (thermooptical) mechanism dominates. As the magnitude of energy release approaches the corresponding magnitude of the heat phase transition (2-3 $\mathrm{J} / \mathrm{sq} . \mathrm{cm})$, the emitted acoustic signal of thermal origin undergoes changes and is combined in a complicated way with the contributions of recoil pulse of the droplet plume, plasma flare and explosive expansion of near surface region of the medium (combinatory mechanism). For energy deposits well above the evaporative threshold evaporative mechanism dominates.

The burst of surface pressure generates a hydroacoustic pulse. It is somewhat alike marine mammals echo-location pulses. The pressure level at a distance of $1 \mathrm{~m}$ from the surface is about 
$0.1 \mathrm{MPa}$, the total acoustic energy transmitted to the ocean medium can amount 1-10 $\mathrm{J}$ or even more.

Among numerous advantageous features of LIUAS the following are most significant for remote sea-bed sensing. Firstly, the signal is of ultra wide band character. Hence the single shot record produces a set of narrow band reverberation responses. In turn, this set of curves can be used for comparison with existing scattering models. Secondly, backscattering coefficient vs angle of incidence for a variety of frequencies can be extracted from these data since LIUAS angular pattern is precisely known. And, finally, high spatial resolution of LIUAS signal and the possibility of laser spot movement thus changing the sensing configuration should be mentioned.

\section{EXPERIMENTAL}

Full scale experiments were conducted in the shallow water region near the Black Sea northen coast in summer time. $\mathrm{CO}_{2}$-laser on board a research vessel was used to launch LIUAS. Laser pulse energy comprised $200 \mathrm{~J}$ and the spot having diameter of $3-5 \mathrm{~cm}$ on the surface could be easily moved within several meters distance. Wide band acoustic signals (both outgoing and scattered) were recorded by $8101 \mathrm{~B} \& \mathrm{~K}$ hydrophones within frequency band of up to $100 \mathrm{kHz}$. The analysis within a broader band (up to $300 \mathrm{kHz}$ ) was provided by a home-made receiver. Various configurations of the system "source-receiver" were used. The 7005 B\&K recorder was used for preliminary storage of reverberation series. The digital records of length of up to $0.5 \mathrm{sec}$ were finally processed by IBM PC using original software. The processing included averaging (if needed), narrow band filtering (relative band $\sim 0.1$ ) and evaluation of reverberation intensity. The results obtained were, firstly, used to estimate the bottom backscattering coefficient. Secondly, these were compared to data obtained from the following model.

\section{THEORY}

Spectrum narrow-band filtering of sea-bed reverberation time-series allows to determine angular structure of the intensity of the sea-bed scattered field for different frequencies. Their analysis, in turn, allows to choose an adequate scattering model and, under appropriate assumptions, makes it possible to evaluate the parameters of the scattering medium.

The following narrow-band reverberation model is presented. Let scattexers be irregularities of the sea-bed surface and those of upper sediment layer. This situation corresponds to the model of high-frequency scattering [2,3]. In this case mean intensity of the narrow-band signal scattered by a sea-bed can be put down in the form

$$
I(\omega, t)=\int I_{0}\left(\theta, \omega, t-2 R_{s} / c\right) m_{s}(\theta, \omega) R_{s}^{-4} d^{2} r
$$

where $\omega=2 \pi f$ is the central angular frequency of a filter, $c$ is the sound velocity in water, $R_{s}=\left(r^{2}+H^{2}\right)^{1 / 2}$ is the distance to an arbitrary point at the scattering surface, given by the incidence angle $\theta=\theta(r), \tan \theta=r / H, H$ is the bottom depth, $t$ is the time delay from the laser shot, $m_{s}(\theta, \omega)$ is the bottom backscattering coefficient, $I_{0}(\theta, \omega, t)$ is the intensity of the emitted signal at a unit distance from the LIUAS. It can be represented in the form

$$
I_{0}(\theta, \omega, t)=I_{1}(\omega) D(\omega, \theta) F(\omega, t)
$$

where $F(\omega, t)$ is the square of emitted signal envelope normalized to its maximum value, $D(\omega, \theta)$ is the frequency-angular pattern of the LIUAS, $I_{1}(\omega)=\max _{t, \theta} I_{0}(\theta, \omega, t)$ is the referent intensity. Going to the integrating over $x=\cos \theta(r)$ in Eq.(1) we obtain 

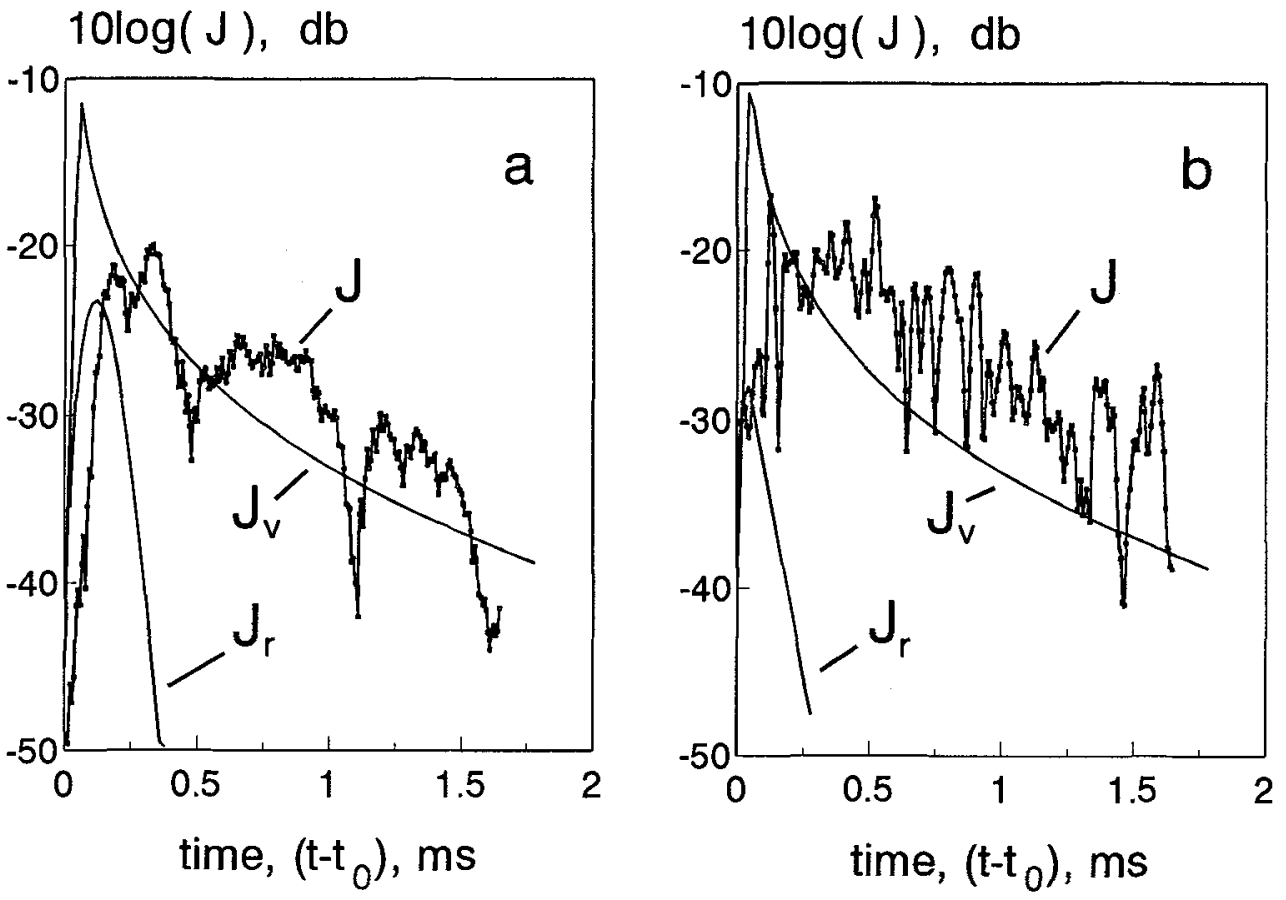

Figure 1: Experimental $J$ and theoretical $\left(J_{r}, J_{v}\right)$ normalized sea-bed reverberation intensities for different frequencies, $16 \mathrm{kHz}$ (a) and $64 \mathrm{kHz}$ (b), $H=4 \mathrm{~m}$. Here $J_{r}$ corresponds to large-scale roughness scattering and $J_{v}$ corresponds to anisotropic volume inhomogeneities scattering.

$$
J_{s}(\omega, t)=4 H^{2} I / I_{1}=8 \pi \int_{t_{0} / t}^{1} D(\omega, x) F\left(\omega, t-t_{0} / x\right) m_{s}(x, \omega) x d x,
$$

at $t>t_{0}$ and $J_{s}=0$ at $t \leq t_{0}$, where $t_{0}=2 \mathrm{H} / \mathrm{c}$ is time delay of the first arrival of scattered signal with respect to laser shot.

For the sake of calculation let us consider the parameters of the emitted LIUAS pulse as follows

$$
\begin{gathered}
D(\omega, x)=x^{n(\omega)}, \\
F(\omega, t)=(t / \tau)^{2} \exp \left[1-(t / \tau)^{2}\right],
\end{gathered}
$$

where $\tau=\tau(\omega)=1 / \Delta \omega, \Delta \omega$ being the filtering band. Function Eq.(4) corresponds to the frequency-"colored" pattern of the near-surface LIUAS radiation, power index $n(\omega)$ increasing as frequency grows up. Function Eq.(5) adequately depicts real LIUAS envelope after it is filtered in the narrow band $\Delta \omega / \omega=0.1$.

The analysis of experimental data at different frequencies has shown that the most adequate model is one of scattering corresponding to frequency independent scattering coefficient. So, let us consider the backscattering coefficient of a sea-bed in the form

$$
\begin{gathered}
m_{s}=m_{r}+m_{v} \\
m=V^{2}\left(8 \pi \tan ^{2} \delta \cos ^{4} \theta\right)^{-1} \exp \left(-\tan ^{2} \theta / 2 \tan ^{2} \delta\right),
\end{gathered}
$$




$$
m_{v}=\alpha \cos H /\left(\sin ^{2} \theta+\eta^{2} \cos ^{2} \theta\right)^{3 / 2},
$$

where $m_{r}$ is the backscattering coefficient of smooth large-scale roughness of bottom relief in Kirchoff approximation [4], $\delta$ is the mean square angle of relief inclination $(\tan \delta \ll 1), V$ is the reflection coefficient at the water-sediment interface, $m_{v}$ is the scattering coefficient of anisotropic volume inhomogeneities of the subsurface sediment layer [5], $\eta$ is an anisotropy factor (i.e. the ratio between vertical and horizontal inhomogeneity scales, $\alpha$ is a constant, independent of frequency. We hence obtain $J_{s}=J_{r}+J_{v}$ which is the normalized reverberation intensity to be compared with the experimental data.

\section{DISCUSSION}

Fig. 1 shows calculated dependencies $J_{r}$ and $J_{v}$ for two frequencies, 16 and $64 \mathrm{kHz}$. We assume that in Eq.(7) $\tan \delta=0.1, V=0.05$ which corresponds to water-silt interface, $\alpha=0.025$ and $\eta=0.1$ in Eq.(8) correspond to the distinctly anisotropic volume inhomogeneities. We use $n=2$ for $f=16 \mathrm{kHz}$ and $n=4$ for $f=64 \mathrm{kHz}$. These plots also show time reverberation records $J$ obtained in experiment. It can be seen that calculated values $J_{v}(t)$ are consistent with experimental records while the qualitative agreement between calculated values of $J_{r}(t)$ and the records cannot be obtained when the parameters of the model presented are changed within judiciously chosen limits. It can be seen also, that relative contribution of scattering by smooth large-scale roughness decreases with the increase of both sound frequency and incident angle (or time at the Figure), while the contribution of volume scattering, on the contrary, increases. Note, that rates of reverberation intensity decrease are identical at both frequencies.

The comparison of experimental and calculated data allows to conclude that the sea-bed reverberation in the region of the experiment was of a volume character rather than of a surface one. Also, it was possible to estimate the backscattering coefficient by sediment inhomogeneities as described by Eq.(8) and their anisotropy factor as well.

\section{CONCLUSION}

In the study presented the emphasis was on the properties of the scattered signal. The followed estimation of sediment parameters can be used for example, for the modelling of sound propagation and scattering in shallow water. Hence the results serve as a contribution to the field of hydroacoustics. Besides, these may be used in oceanology, thorough marine sounding itself being our further aim.

\section{References}

[1] Egerev S.V., Lyamshev L.M. and Naugol'nykh K.A., Sov. Phys. Acoust. 36 (1990) 452-456.

[2] Ivakin A.N., "Sound scattering from ocean bottom: theory and experiment", European Conference on Underwater Acoustics, Luxembourg 14-18 September 1992 (Elsevier Applied Science, 1992) pp.521-524.

[3] Ivakin A.N. and Lysanov Yu.P., Sov. Phys. Acoust. 27 (1981) 212-215.

[4] Brekhovskikh L.M. and Lysanov Yu.P., Fundamentals of Ocean Acoustics (Springer-Verlag, Berlin, New-York, 1990) pp.270.

[5] Ivakin A.N., Okeanologia 21 (1981) 42-44. 